\title{
Molecular Detection of Feline Herpesvirus by Means Polymerase Chain Reaction
}

\author{
Paulina Macías and Carlos Navarro* \\ Department of Animal Preventive Medicine, Faculty of Veterinary Medicine and Animal Sciences, University of Chile
}

Submission: November 09, 2018; Published: November 30, 2018

*Corresponding author: Carlos Navarro, Department of Animal Preventive Medicine, Faculty of Veterinary Medicine and Animal Sciences, University of Chile, Chile

\section{Abstract}

In the Feline Respiratory Complex, the Feline herpesvirus is a main etiological agent with worldwide distribution and affect domestic cats causing ocular and upper respiratory tract lesions. In Chile, only clinical diagnostic is used, unlike use several techniques in other countries and Polymerase Chain Reaction (PCR) is the most recommended with best performing of them. For implemented an effective diagnostic method in our country, we select cats under one-year-old with clinical signs suggest feline herpesvirus infection, to detect thymidine kinase protein gen of herpesvirus, highly specific and conserved, by a nested PCR and subsequent determination of nucleotide identity percentage respect GenBank ${ }^{\circledR}$, genomic database.

As a result, we obtained a high detection rate (100 percent of samples and positive control), and a 92 percent of nucleotide identity in comparison with de genomic database GenBank $®$ (accesion number E12463.1), proving that correspond to feline herpesvirus. Thus, achieved described an effective diagnostic method to be used as a complementation of clinical diagnostic.

Keywords: Feline herpesvirus; Nested PCR; Thymidine kinase; Feline herpesvirus diagnosis

Abbreviations: FHV: Feline Herpes Virus; Kb: kilobases; AV: Viral Isolation; IFI: Indirect Immunofluorescence; SN: Seroneutralization; ELISA: Enzyme-Linked Immune Sorbent Assay; PCR: Polymerase Chain Reaction

\section{Introduction}

Feline herpes virus type 1 (FHV-1) was isolated in 1957 by Crandell and Maurer [1]. It is a DNA virus linear double strand containing approximately 134 kilobases (kb), subdivided into long and short components of 104 and $30 \mathrm{~kb}$ respectively [2,3], which is immersed in a capsid of icosahedral symmetry constituted by 162 capsomeres. Surrounding the capsid is the tegument, an amorphous matrix that contains globular proteins with enzymatic activity. Outside the tegument is a pleomorphic lipoprotein envelope that presents spicules, many of which are responsible for inducing an immune response in the host [4]. FHV-1 is widely distributed in the world, with approximately $90 \%$ of cats being seropositive to the virus $[5,6]$. Only one serotype is known, which differs in virulence depending on the strain [7]. In adult cats it causes high morbidity and low mortality, whereas in small cats it can generate a mortality of $60 \%$ [8].

FHV-1 belongs to the order Herpesvirales, Herpesviridae family, Alphaherpesvirinae subfamily and Varicellovirus genus [9]. The viruses of this subfamily are characterized by being highly species-specific, have a short replicative cycle of 24 hours, labile to the environment and common disinfectants, possess a marked tropism to the epithelia and generate latency in neural tissue $[10,11]$. The domestic cat is the main host of FHV-1. The route of entry of the virus is nasal, oral or conjunctival, causing multifocal epithelial necrosis, with neutrophilic infiltration and inflammation in conjunctiva, pharynx, trachea, bronchi to bronchioles [7]. The lesions are caused by two mechanisms: direct, product of viral replication that leads to cytolysis, and indirect, through the action of inflammatory cells $[6,11]$. It does not cause viremia, except in neonates or in hypothermic puppies, since viral replication occurs preferably at low temperatures. Viral excretion begins 24 hours after the infection occurs, and is maintained for one to three weeks, while the acute clinical picture resolves within 10 to 14 days in immunocompetent cats [12].

This virus causes latency in the trigeminal ganglion mainly and $80 \%$ of infected cats become carriers for life $[13,14]$. The reactivation of the virus can be induced experimentally by treatment with glucocorticoids. However, there are other factors that can reactivate the virus through stress such as: transfer to a new environment (18\% of cases), childbirth and breastfeeding (in $40 \%$ of cases) [7]. In this way, small cats acquire the virus very early (Conjuntitivis neonatorum) [8]. The development of the disease depends on the level of maternal antibodies that they possess, which provide passive immunity via colostral during the first weeks of life. When high levels of antibodies are present, kittens are protected from the disease and develop a subclinical infection that may end up becoming latent. When there are not enough 
maternal antibodies, the clinical disease develops. Unfortunately, herpes virus infection does not provide a strong active immune response, so infected females do not always have adequate antibodies. Therefore, the immune response protects against the disease, but not against the infection [7]. The prevalence of the virus can range between $1-20 \%$ depending on the size of the cat population. In catteries, the risks are greater, product of the high animal density and the bad hygienic conditions $[7,15]$.

\section{Clinical Signs}

The clinical illness can be divided into: Classic acute illness: very severe in cats under one year of age. They present fever, rhinitis, conjunctivitis, corneal ulcers both superficial and deep, abundant mucopurulent secretion, both nasal and ocular and recurrent sneezing. This leads to depression and anorexia. Atypical acute illness: they present oral and nasal ulcers, dermatitis with crusted lesions or pneumonia. Chronic illness: they present stromal keratitis with corneal edema, vascularization, corneal ulcers can evolve to corneal sequestration, blindness or a chronic rhinosinusitis with nasal discharge and sneezing for life.

Some adult cats may develop lesions due to viral reactivation, which is called recrudescence. They may present clinical signs associated with both an acute condition and may progress to a chronic condition [7,15]. There is often coinfection with feline calicivirus and/or Chlamydophila felis, Bordetella bronchiseptica, Mycoplasma sp, Staphylococcus sp, or Escherichia coli, causing a multi-causal feline respiratory syndrome. There are vaccines against FHV-1, but it has been shown that they do not provide complete protection in all cats, especially in places with high animal density, where viral load is high $[10,16]$.

\section{Diagnosis}

The primary infection with FHV-1 is so aggressive that the clinical diagnosis is simple. In contrast, in adults the clinical signs in its chronic phase are mild and diverse, which makes viral identification necessary [17]. Among the differential diagnoses of an individual with rhinosinusitis, are: viral (Herpes, Calicivirus), bacterial (Chlamydophila felis, Bordetella bronchiseptica, Mycoplasma sp. as primary agent), fungal (Cryptococcus neoformans, Aspergillus spp, Histoplasma capsulatum, Blastomyces dermatitidis), parasitic (Capillaria aerophila, Syngamus ierey), foreign bodies, allergies, dental alterations, polyps, neoplasms (adenocarcinoma, for example), lymphoplasmacytic rhinosinusitis and trauma [18]. Similar is the case of a cat with conjunctivitis, whose differential diagnoses are: viral (Herpes, Calicivirus, feline immunodeficiency virus, feline viral leukemia), bacterial (Chlamydophila felis, Bartonella sp., Mycoplasma sp.), Food allergy, atopy, traumatic (eyelash changes, irritant factors, etc.) [17]. There are several diagnostic methods such as: viral isolation $(\mathrm{AV})$, indirect immunofluorescence (IFI), seroneutralization (SN), enzyme-linked immune sorbent assay (ELISA) and Polymerase Chain Reaction (PCR). Compared with these, PCR is 25\% more sensitive than viral isolation from conjunctival scrapings, presumably due to an inactivation of viral infectivity because the envelope is easily destroyed by transport, freezing, enzymes or antibodies present in saliva or tea $[8,19]$. In addition, it does not need the virus to be viable and its results are not altered using vital dyes such as fluorescein (necessary to diagnose corneal ulcers).

Unfortunately, it is not known if individuals vaccinated against HVF-1 produce false positives when sampled and subjected to PCR. A study carried out in 2011 concludes that after vaccinating healthy individuals, both nasally and parenterally, a low percentage of positives is obtained when using the PCR method. However, only two vaccines were studied, so future research is needed to clarify this question [20]. As for other diagnostic techniques, such as AV and IFI, they have a high sensitivity in cases where the clinical picture is acute, but not in chronic or recrudescent cases [8]. Another disadvantage that has IFI is to be a very subjective technique to depend on the experience of the operator, and without good results, due to the low amount of viral antigen and interference by the immune response, either humoral or mediated by inflammatory cells, which increases the possibility of false negatives [21]. In addition, IFI, SN and AV are procedures that require a longer time and are expensive compared to PCR.

For these reasons, PCR is the method of choice for the detection of FHV-1. Variations of conventional PCR have been used: nested or in real time, the latter being the most modern and with the capacity to provide additional information, for example: a high number of copies suggests active replication, whereas a low number indicates an infection latent with FHV-1. The samples used to perform the PCR are diverse and have in common the integration of different tissues where FHV-1 latency. The main detection sites are: trigeminal ganglion, optic nerve, olfactory bulbs, cornea and nasal turbines. Less frequently, the virus is detected in: salivary and lacrimal glands, oral cavity, tonsils and conjunctiva. The latter are more used since their extraction is not necessarily post-mortar, but by scraping, brushing or swabbing tissue. The most widely used technique worldwide now amplifies a segment of the gene that codes for the protein Thymidine Kinase [2]. It has the characteristic of being highly divergent in its amino acid sequence between the different species of herpes viruses, and extremely conserved among the isolates of FHV-1 [2]. Initially, conventional PCR was used, achieving detection rates ranging from $25-30 \%[8,10,19]$. Subsequently, a nested PCR was started $[8,22]$, thus achieving detection rates of $54 \%$. It is described that it is 10 times more sensitive than conventional PCR [22].

Other study [23] compared six PCR protocols that use the Thymidine Kinase protein gene for the identification of FHV-1 and analyzed its viral detection rate against a study population affected by the virus [11]. In conclusion, it can be concluded that the PCR that obtained the best results with a viral detection rate of $89 \%$ [22], which included a nested PCR (n-PCR). say, he used two pairs of different primers in sequential amplification reactions. The first pair of primers amplifies a DNA fragment which is then used as a template in a second reaction. The pair of primers used in the second round of amplification, verifies the specificity of the product obtained in the first PCR and the transfer of the first 
product obtained to a new reaction mixture, has the useful effect of dilution of the possible inhibitors that may exist in the original sample [4]. Thus, the work of Stiles and colleagues is the main reference in this report to achieve the implementation of a PCR (in this case nested) that seeks to detect the Thymidine Kinase gene from feline herpes virus, to complement the clinical diagnosis of the virus, since there is currently no molecular diagnostic test for this specific virus in Chile.

\section{Material and Methods}

Samples. Samples from 11 short-haired domestic cats under one year of age were used, without previous vaccinations, who presented the clinical signs of: conjunctivitis uni or bilateral, mucopurulent nasal and ocular discharge, paroxysmal sneezing, blepharospasm and/or corneal ulcer, typical of acute infection with FHV-1. Subsequently, each of them underwent a swab with a dry sterile tórula on the ventral conjunctival mucosa of one or both affected eyes. The samples were kept refrigerated at $4{ }^{\circ} \mathrm{C}$ for 3 weeks, before the content of the swab was homogenized in a tube with $200 \mathrm{uL}$ of nucleated free water (Winkler $®$ ), previously numbered, using a Heidolph $\AA$ tube agitator. The Feligen ${ }^{\circledR}$ lyophilized triple-feline attenuated vaccine from the Virbac Laboratory was used as a positive control, which was resuspended in $500 \mathrm{uL}$ of the diluent recommended by the manufacturer. As a negative control, a swab with a sterile swab was obtained from each eye of a cat clinically free of feline herpes since childhood and without vaccinations.

\section{Detection of the Thymidine Kinase (TK) gene in FHV- 1 by $\mathrm{n}-\mathrm{PCR}$}

\section{Nested PCR}

The primers used to detect the gene Thymidine Kinase of FHV-1 in this first reaction (PCR1) amplify a fragment of 383 base pairs (bp) (Reubel et al., 1993)) and were commissioned to the company Bioscan $®$ for its preparation: FHV-1A 5' -GCATTTACATA GATGGTGCCT - 3' and 5' -ATATCTTGCG AGTGGGAAACAG - 3'. In the second reaction (PCR2), a second pair of primers was used to amplify an internal segment of DNA obtained in PCR1 and with a size of 224 pairs of DNA. bases (Stiles et al., 1997): FHV-1B 35' CTTAC TACTTCCCAGAACC - 3' and 5' - GTTCC T CACATACAACTTTC -3 .

\section{Reaction Mixture}

15uL of the 2X PCR master mix commercial kit (Taq DNA polymerase, $\mathrm{MgCl} 2$ and the deoxyribonucleotides trifostatos), $1 \mathrm{uL}$ of sample and $5 \mathrm{uL}$ of each specific splitter, in a final volume of 26uL were used. b) DNA amplification [22]: Both the first and the second reaction of the nested PCR (PCR1 and PCR2) are governed by the same amplification protocol: after the initial denaturation at $94^{\circ} \mathrm{C}$ for 4 minutes, a PCR sequence of 35 cycles (denaturation: $94{ }^{\circ} \mathrm{C}$ for 1 minute, alignment: $55^{\circ} \mathrm{C}$ for 1 minute, extension: $72{ }^{\circ} \mathrm{C}$ for 1 minute). Subsequently, a final elongation stage at $72^{\circ} \mathrm{C}$ for 8 minutes.

\section{Visualization of Amplified Products}

It was performed by electrophoresis in $2 \%$ agarose gel (Winkler (®) in Tris-borate buffer ( $90 \mathrm{Mm}$ Tris-borate, $10 \mathrm{mM}$ EDTA) as solvent. An aliquot of $5 \mu \mathrm{L}$ of this mixture was deposited in the respective well of the gel. Electrophoresis was carried out at $90 \mathrm{~V}$ for 45 minutes. As a molecular size marker, a standard containing DNA fragments between 100 and 1000 bp (Fermentas $囚$ ) was used. After electrophoresis, the gel was incubated in ethidium bromide $(0.5 \mu \mathrm{g} / \mathrm{mL}$ ) (Fermelo $\AA$ ) for 45 minutes and then placed in a transilluminator of ultraviolet light (Transiluminator UVP $₫$ ) and photographed.

\section{Biosafety Measures}

The laboratory work was carried out in accordance with the biosafety levels established for microbiology and animal virology laboratories, such as the use of clean material, correct waste disposal and the use of a closed apron, and gloves in practical work. The process of visualizing the amplified product involves the use of ethidium bromide and a UV light transilluminator. Due to this, at the time of visualizing the gel an acrylic plate and glasses with UV filter were used. Subsequently, the elimination of the gel submerged in ethidium bromide contemplated its incineration, since the chemical compound mentioned has -among othersmutagenic properties.

\section{Determination of Percentage of Nucleotide Identity with Respect to Data from Genbank ${ }^{\circledR}$. Sequencing}

After being submitted to the first PCR, two samples were sent to the Sequencing Center of the company Genytec Ltd. according to their requirements, which performed the purification of the amplified. The sequences were made using the Big Dye Terminator Kit, Applied Biosystems, and an ABI PRISM 310 computer was used to read them. Genetic Analyzer (Genytec specifications).

\section{Analysis}

Using the open-access online program Clustal $\Omega$, the sequences delivered by Genytec Ltd. were aligned to achieve a consensus sequence (PMS) that was then compared with the fragment of the Thymidine Kinase from the feline herpes virus of GenBank® (access number E12463 .1), thus establishing the percentage of nucleoid identity. By way of comparison, the results of Genytec Ltd. were also incorporated into the BLAST Online Access Program.

\section{Results}

\section{Nested PCR}

When performing PCR1 (Figure 1) it was observed that in 3 of the 13 samples a DNA fragment of a size close to 400 base pairs was visualized, achieving a visible band in the clear and wide gel. However, in the positive control (vaccine) a visible band was not obtained. On the other hand, in the PCR2 products (Figure 2) it was possible to observe in all the samples and positive control a fragment of DNA of molecular size around 200 base pairs, achieving a band of different quality of display. These samples can be diagnosed as positive. 


\section{Journal of Dairy \& Veterinary Sciences}

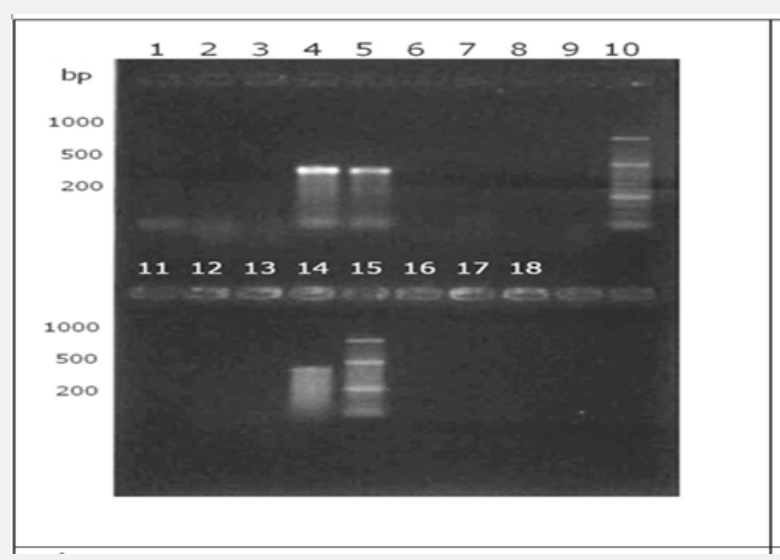

Figure 1: Detection of the Timidin Kinase gene by nested PCR. Display of PCR1 results. Lane 1-9: samples 1-9, lane 10: molecular size marker (50-1000 bp, Fermentas $\left.{ }^{\circledR}\right)$, lane 11-14: samples 10-13, lane 15: molecular size marker, lane 16: positive control (vaccine), lane 17 and 18: negative control.

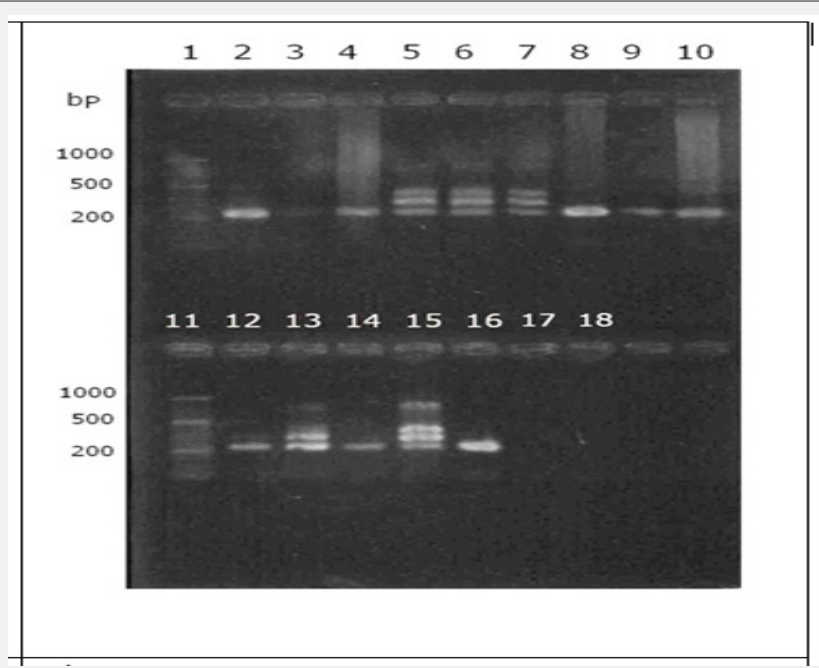

Figure 2: Detection of the Timidin Kinase gene by nested PCR. Display of PCR2 results. Lane 1: Molecular size marker (50-1000 bp Fermentas $($ ) , lane 2-10: samples 1-9, lane 11: molecular size marker, lane 12-15: samples 10-13, lane 16: positive control (vaccine), lane 17-18: negative control.

\section{Analysis of the Sequenced Fragment}

\begin{tabular}{|c|c|c|c|c|c|c|c|c|}
\hline \multicolumn{9}{|c|}{ 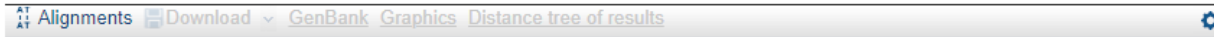 } \\
\hline & & Description & $\begin{array}{l}\text { Max } \\
\text { score }\end{array}$ & $\begin{array}{l}\text { Total } \\
\text { score }\end{array}$ & $\begin{array}{l}\text { Query } \\
\text { cover }\end{array}$ & $\begin{array}{c}E \\
\text { value }\end{array}$ & Ident & Accession \\
\hline$\square$ & Felid alphaherpesvirus 1 strain KANS 02, complete genome & & 676 & 676 & $74 \%$ & 0.0 & $99 \%$ & $\underline{\mathrm{MH} 070348.1}$ \\
\hline$\square$ & Felid alphaherpesvirus 1 strain MILW 11, complete genome & & 676 & 676 & $74 \%$ & 0.0 & $99 \%$ & $\underline{\mathrm{MH} 070347.1}$ \\
\hline$\square$ & Felid alphaherpesvirus 1 strain MILW 09 , complete genome & & 676 & 676 & $74 \%$ & 0.0 & $99 \%$ & $\underline{\mathrm{MH} 070346.1}$ \\
\hline$\square$ & Felid alphaherpesvirus 1 strain WASH 03 , complete genome & & 676 & 676 & $74 \%$ & 0.0 & $99 \%$ & $\underline{\mathrm{MH} 070345.1}$ \\
\hline$\square$ & Felid alphaherpesvirus 1 strain PHIL 10, complete genome & & 676 & 676 & $74 \%$ & 0.0 & $99 \%$ & $\underline{\mathrm{MH} 070344.1}$ \\
\hline$\square$ & Felid alphaherpesvirus 1 strain NEWY 03 , complete genome & & 676 & 676 & $74 \%$ & 0.0 & $99 \%$ & $\underline{\mathrm{MH} 070343.1}$ \\
\hline$\square$ & Felid alphaherpesvirus 1 strain MILW 02 , complete genome & & 676 & 676 & $74 \%$ & 0.0 & $99 \%$ & $\underline{\mathrm{MH} 070342.1}$ \\
\hline$\square$ & Felid alphaherpesvirus 1 strain FLOR 04. complete genome & & 676 & 676 & $74 \%$ & 0.0 & $99 \%$ & $\underline{\text { MH070341.1 }}$ \\
\hline$\square$ & Felid alphaherpesvirus 1 strain PEEBLES 1 complete genome & & 676 & 676 & $74 \%$ & 0.0 & $99 \%$ & $\underline{\mathrm{MH} 070340.1}$ \\
\hline$\square$ & Felid alphaherpesvirus 1 strain CALI 14, complete genome & & 676 & 676 & $74 \%$ & 0.0 & $99 \%$ & $\underline{\mathrm{MH} 070339.1}$ \\
\hline$\square$ & Felid alphaherpesvirus 1 strain MILW 12, complete genome & & 676 & 676 & $74 \%$ & 0.0 & $99 \%$ & $\underline{\mathrm{MH} 070338.1}$ \\
\hline$\square$ & Felid alphaherpesvirus 1 strain KANS 04 , complete genome & & 676 & 676 & $74 \%$ & 0.0 & $99 \%$ & $\underline{\mathrm{MH} 070337.1}$ \\
\hline$\square$ & Felid alphaherpesvirus 1 strain PHIL 01, complete genome & & 676 & 676 & $74 \%$ & 0.0 & $99 \%$ & MH070336.1 \\
\hline
\end{tabular}

Figure 3: Sequences producing significant alignments. 
The obtained amplifications were successfully sequenced. Subsequently, they were aligned to obtain a consensus sequence (PMS) and thus compare with with the GenBank ${ }^{\circledR}$ data for FHV1 using the BLAST program (Figure $2 \& 3$ ), obtaining an average value of $99 \%$ nucleotide identity.

\section{Discussion}

Based on the results obtained in this study, the following reflections can be made: in PCR2 all samples were positive, so it is thought that there was indeed amplification when using primers 1 and 2 in PCR1, but because after PCR1 the samples and the positive control had a low number of amplicons, they did not generate a visible band in the UV transilluminator. The results of the PCR2 are explained by the condition of being a nested PCR, where in the second reaction it was sought to amplify a segment immersed in the amplicon resulting from the first PCR, increasing the sensitivity and specificity of the test.

The positive samples in PCR1 corresponded to individuals who had severe clinical signs, with blepharospasm in all and epistaxis in sample 5, which suggests that the greater the severity of clinical signs, the greater the amount of virus excreted, which entails to an evident visualization of the band in the UV transilluminator. In support of this premise, in the PCR2 results it was observed that sample 1 and 2 (belonging to the same cat) were positive, but with differences in their sharpness. The band visualized in sample 1 was evidently more extensive and marked than sample 2, which was less visible. This was obtained from the right eye, which presented greater clinical severity with respect to the left eye, from which sample number 2 was obtained. This same situation is repeated in samples 11 and 12 , but of a different cat. This explains that with 11 cats under study, 13 samples were obtained.

In PCR2, it was possible to appreciate that the primers used are specific, since in 8 out of 13 samples they generated a single band of $224 \mathrm{bp}$. It should be noted that these 8 samples did not present visible bands after visualization in the UV transilluminator after PCR1. In contrast, in the remaining 5 samples, 3 bands of different molecular size were observed, which, based on the molecular marker, would be approximately 400, 300 and $200 \mathrm{bp}$. Bands of 400 and $200 \mathrm{bp}$ would correspond to the amplicon obtained in PCR1 (383 bp) and that obtained in the PCR2 (224 bp) respectively. The appearance of a third band of $300 \mathrm{bp}$ could be explained because the samples that were used for PCR2 corresponded to the resulting solution after PCR1, causing that in the second reaction were the four used starters, which are governed under the same temperature protocol in the thermal cycler, that is, they are specifically linked to a mold thread at the same time, generating four possibilities of amplicons depending on their association. The combinations of starters according to the direction of nucleotide synthesis (from 5 'to 3 ') would be: 1-2 (383 bp), 3-4 (224 bp), 1-4 (311 bp) and 2-3 (295 bp). Since the last two would have a similar molecular size, it is explained that in the UV transilluminator only 3 bands are appreciated instead of 4 .

The five samples in which the 3 previously described bands were observed ( 3 of which were the only positive ones in the
PCR1), correspond to the cats that were more severe in their clinical signs comparatively with the rest of the individuals in the study. If these cats would excrete a greater amount of virus, the explanation for the appearance of the 3 bands only in these cases could be because having a large amount of viral DNA in a sample and then carrying out the PCR, visualize all the amplicons formed. On the other hand, when there is scarce viral DNA, only the most amplified segment in the UV transilluminator can be observed, corresponding to that obtained thanks to primers 3 and 4, added in the second PCR reaction. For this reason, it is proposed that in those samples when the nested PCR is visualized these 3 bands, repeat the PCR2, considering that instead of using $1 \mathrm{uL}$ of sample, take $0.1-0.5 \mathrm{uL}$.

It is important to point out that this phenomenon is not observed in any of the samples of the publication that is taken as reference in this report [22]. A possible explanation could be due to the characteristics of the cats sampled in both studies, since at present only cats under one year were selected. On the other hand, Stiles et al. [22] considered felines of all ages, mainly adults, whose clinical picture was characterized by lower severity, and therefore lower viral excretion. In relation to the high detection rate, it is important to note that 9 of the cats sampled ( 9 out of 11) belong to the same feline refuge, where they have shared for 2 weeks a small cage. Knowing the high morbidity of FHV-1 there is a high possibility that all of them are infected.

In this report it could also be determined that, consequently with the results obtained in a recent study [23], three weeks of refrigeration did not influence the detection of FHV-1 by PCR, given that all the analyzed samples were positive. Compared with other PCR protocols worldwide, the implemented in this work reached a high detection rate. A study conducted at the University of California in 2005 [23], compared the "performance" (relative detection rate, and the minimum detected by dilutions) of 6 PCR protocols described by different researchers who have in common to detect the gene of the Thymidine Kinase, which were the most used in laboratories. They differ in the specific segment to be amplified from the gene, the type of PCR (conventional or nested), the starters, temperature and number of cycles in the thermal cycler. The results show detection rates from 29 to $86 \%$. It should be noted that the samples corresponded to cats suspected of FHV1 and it was not verified whether they were indeed infected by other diagnostic tests, such as viral isolation.

Among the protocols that entered the study (Reubel et al., 1993) that uses the same PCR protocol and PCR1 primers of this title memory, but performs only a conventional PCR, obtaining a $29 \%$ rate of detection. In another study [22], another successive PCR with different primers was added to the Reubel conventional PCR, that is, it performed a nested PCR, obtaining a rate of 54\% detection. Years later, it was replicated by another researcher [11] who obtained $86 \%$, in contrast to the present study that obtained a $100 \%$ detection rate. The cats that entered the different studies [22-24] had a wide range of age (7 months to 15 years) and were suspected of infection with FHV-1, both acute and chronic. These 
variables are the ones that would explain that, despite being the same nested PCR protocol, different detection rates are obtained.

That is why in this title report we sought to minimize these variables, focusing on the detection rate in individuals under one year of acute infection with FHV-1, therefore, it is proposed in later studies to detect this virus in adult cats that are studying the chronic phase of the disease, with the signology that is associated with such clinical picture. The high percentage of nucleotide identity obtained shows that indeed the amplified DNA fragment corresponds to the gene of the protein Thymidine Kinase of the feline herpes virus, and not to another herpes virus, although it is known that one of the characteristics of the viral Alpha herpes virus family is to be highly species-specific.

\section{Conclusion}

In this report, the molecular diagnosis of FHV-1 is first described in Chile by the detection of the Timidin Kinase protein gene by the nested PCR technique, which has a high detection rate $(100 \%)$ in cats. infected children under one year of age, who are in the acute stage. The percentage of nucleotide identity obtained of $92 \%$ allows to affirm that the detected virus corresponds to the feline herpes virus.

\section{References}

1. Thai S, Niikura M, Cheng H, Kruger J, Wise A, Maes R (2010) Complete genomic sequence and an infectious BAC clone of feline herpesvirus-1. Virology 401: 215-227.

2. Nunberg J, Wright D, Cole G (1989) Identification of the timidine kinase gene of feline herpesvirus: use of degenerate oligonucleotides in the polymerase chain reaction to isolate herpesvirus gene homologs. J Virol 63: 3240-3249.

3. ICTV (2018) International Committee on Taxonomy of Viruses.

4. Murphy F, Gibbs E, Horzinek M, Studdert M (2000) Veterinary Virology, ( $3^{\text {rd }}$ edn), Academia Press, New York, United States, pp. 629.

5. Sandmeyer L, Waldner C, Bauer B, Wen X, Bienzlr D (2010) Comparison of polymerase chain reaction tests for diagnosis of feline herpesvirus, Chlamydophila felis, and Mycoplasma spp. infection in cats with ocular disease in Canada. Can Vet J 51: 629-633.

6. Maes R (2012) Felid herpesvirus type 1 infection in cats: a natural host model for alphaherpesvirus pathogenesis. ISRN Vet Sci pp. 495830.

7. Thiry E, Addie D, Belák S (2009) Feline herpesvirus infection: ABCD guidelines on prevention and Management. J Feline Med Surg 11: $547-$ 555.

8. Burgesser K, Hotaling S, Schiebel A (1999) Comparison of PCR, virus isolation and direct fluorescent antibody staining in the detection of naturally occurring feline herpesvirus infections. J Vet Diagn Invest 11: 122-126.

9. Davison A, Eberle R, Ehlers B (2009) The order Herpesvirales. Arch Virol 154: 171-177.
10. Sykes J, Browning G, Anderson G (1997) Differential sensitivity of culture and the polymerase chain reaction for detection of feline herpesvirus 1 en vaccinated and unvaccinated cats. Archiv Virol 142: 65-74.

11. Maggs D (2001) Update on the diagnosis and management of feline herpesvirus-1 infection. En: AUGUST, J. Consultations in Feline Internal Medicine. ( $4^{\text {th }}$ edn), United States, Editorial Saunders pp.151-161.

12. Reagan KL, Hawley JR, Lappin MR (2014) Concurrent administration of an intranasal vaccine containing feline herpesvirus-1 (FHV-1) with a parenteral vaccine containing FHV-1 is superior to parenteral vaccination alone in an acute FHV-1 challenge model. Vet J 201: 202226.

13. Weigler C, Babineau C, Sherry B (1997) High sensitivity polymerase chain reaction assay for active and latent feline herpesvirus infectations in domestic cats. Vet Rec 140: 335-338.

14. Cleator GM, Klapper PE, Herpes simplex (2000) In: Zuckerman AJ, Banatuala JE, Pattison JR, editors. Principles and Practice of Clinical Virology. ( $4^{\text {th }}$ edn), Chichester: John Wiley and Sons p. 24-45.

15. Najafi H, Madadgar O, Jamshidi S, Arash Ghalyanchi Langeroudi AG, Lemraski MD (2014) Molecular and clinical study on prevalence of feline herpesvirus type 1 and calicivirus in correlation with feline leukemia and immunodeficiency viruses. Vet Res For 5: 255 - 261.

16. Berger A, Willi B, Meli ML, Boretti F, Hartnack S, et al. (2015) Feline calicivirus and other respiratory pathogens in cats with Feline calicivirus-related symptoms and in clinically healthy cats in Switzerland. BMC Vet Res.11: 282.

17. Aroch I, Ofri O, Sutton G (2008) Ocular manifestations of systemic diseases. En: MAGGS, D.; MILLER, P.; OFRI, R. Slatter's, fundamentals of veterinary ophtalmology. ( $4^{\text {th }}$ edn), United Stated, Editorial Saunders, pp. 376-383.

18. Ruiz R (2005) Enfermedades de los senos nasales en gatos. En: MONTOYA, J.A. Enfermedades respiratorias en pequeños animals. Editorial Inter-Medica, Buenos aires, Argentina. p. 89.

19. Reubel G, Ramos R, Hickman M (1993) Detection of active and latent feline herpesvirus 1 infections using the polymerase chain reaction. Arch Virol 132: 409-420.

20. Ruch-Gallie R, Veir J, Hawley J, Lappin M (2011) Results of molecular diagnostic assays targeting feline herpesvirus- 1 and feline calicivirus in adult cats administered modified live vaccines. J Felin Med Surg 13: 541-545.

21. Westermeyer H, Kado-Fong H, Maggs D (2008) Effects of sampling instrument and processing technique on DNA yield and detection rate of feline herpesvirus-1 via polymerase chain reaction assay. Am J Vet Res 69: 811-817.

22. Stiles J, Mcdermott M, Willis M (1997) Comparison of nested polymerase chain reaction, virus isolation, and fluorescent antibody testing for identifying feline herpesvirus in cats with conjuntivitis. Am J Vet Res 58: 804-807.

23. Maggs D, Clarke H (2005) Relative sensitivity of the polymerase chain reaction assays used for detection of feline herpesvirus and commercial vaccines. Am J Vet Res 66: 1550-1555.

24. Clarke H, Kado-Fong H, Maggs D (2006) Effects of temperature and time in transit on polymerase chain reaction detection of feline herpesvirus DNA. J Vet Diagn Invest 18: 388-391. 
